\title{
How the unsolved problem of finding the Healthy Life Expectancy (HLE) in the far past was resolved: The case of Sweden (1751-2016) with forecasts to 2060 and comparisons with HALE
}

\author{
Christos H Skiadas $^{1}$ and Charilaos Skiadas ${ }^{2}$ \\ ${ }^{1}$ ManLab, Technical University of Crete, Chania, Greece \\ (E-mail: skiadas@cmsim.net) \\ ${ }^{2}$ Department of Mathematics and Computer Science, Hanover College, Indiana, \\ USA \\ (E-mail: skiadas@hanover.edu )
}

\begin{abstract}
Healthy Life Expectancy (HLE) estimates are achieved after systematic work of a large group of researchers all over the world during last decades. The most successful estimate was termed as HALE and is provided by the World Health Organization (WHO) in the related website. Having established a methodology of data collection and handling the HLE can be estimated and provided to researchers and policy makers.

However, it remains an unexplored period of the last few centuries where, LE data exists along with the appropriate life tables, but not enough information for HLE estimates is collected and stored. The problem is now solved following a methodology of estimating the HLE from the life tables after the Healthy Life Years Lost (HLYL) estimation. Our methodology on a Direct HLYL estimation from Life Tables, is tested and verified via a series of additional methods including a Weibull parameter test, a Gompertz parameter alternative and of course a comparison with HALE estimates from WHO. The complete
\end{abstract}

September 7, 2020 
methodology and estimation methods are published in the book on "Demography of Population Health, Aging and Health Expenditures" of Volume 50 of the Springer Series on Demographic Methods and Population Analysis.

https://www.springer.com/gp/book/9783030446949, https://doi.org/10.1007/978-3-030-44695-6

Keywords: Life Expectancy, Healthy Life Expectancy, HALE, Logistic model, forecasts, Life Tables

\section{Life Expectancy and Healthy Life Expectancy estimates}

Based on the data series from 1900 to 2016 for males and females in Sweden, estimates until 2016 and forecasts to 2060 are done. The Logistic model is fitted to data series to calculate the three parameters of the model. Then forecasts to 2060 are done. For fitting and long range forecasts the Logistic Model is selected.

1900 was a milestone in health improvement in many countries. As Jan Sundin and Sam Willner (2007) report for Sweden:

"Some diagnoses, such as smallpox, ague, dysentery and cholera, had essentially disappeared in 1900, whilst diphtheria, whooping cough and measles continued to be a real, albeit drastically reduced, threat during childhood."

According to Sunding and Willner the decades before 1900 several important key-points have been done in Sweden including:

-1862 Local government reform: Establishment of the Landsting (county councils, who take over the responsibility for hospitals). -1878 National Medical Board (Medicinalstyrelsen) is founded. -1890 Chief provincial doctor appointed in every county. -1891 First sanatorium for lung disease sufferers in Sweden is opened. 
The development of the first healthcare system of modern history, started with policies introduced by the Otto von Bismarck's social legislation (1883-1911). The introduction of such systems in many countries came after important discoveries from scientists as Pasteur and Chamberland in France, Von Behring in Germany, Kitasato from Japan, Descombey from France and many others. The 1901 Nobel Prize in Physiology or Medicine, the first one in that field, awarded to Von Behring for his discovery of a diphtheria antitoxin.

It looks like the health care systems and methodologies already set in 1900 follow a rather systematic trend until today. See figure 1 where Life Expectancy (LE) data series is provided by the Human Mortality Database (HMD) and Healthy Life Expectancy (HLE) is estimated with our Direct methodology (Skiadas \& Skiadas 2018a,b and 2020a,b,c). The LE series from 1751-1875 is strongly fluctuating mainly due to health causes. The fluctuations become smaller after this period with a clear stabilization from 1900 until now except the strong declining during the 1918 influenza pandemic followed with a fast recovery later on. The period starting from 1950 is followed with a rather smooth trend as a result of the improvement of the health systems structure, financing, technology and pharmaceutical discoveries and production. 


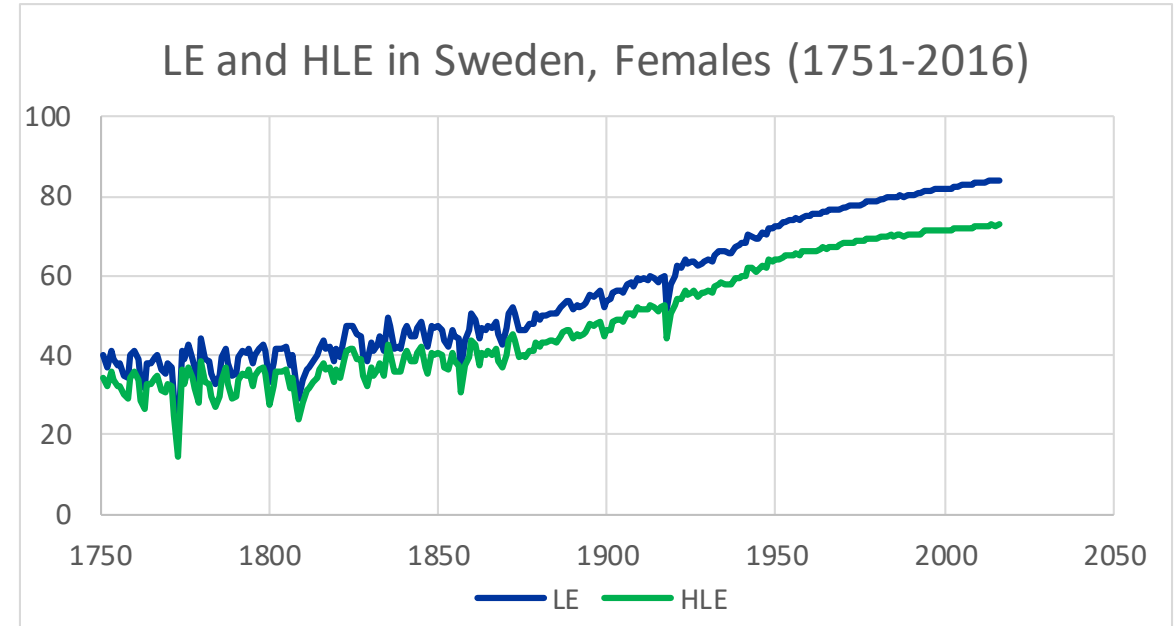

Fig. 1. Life Expectancy (LE) and Healthy Life Expectancy (HLE) in Sweden, females (1751-2016).

Healthy Life Years Lost (HLYL) in Sweden, Females

(1751-2016)

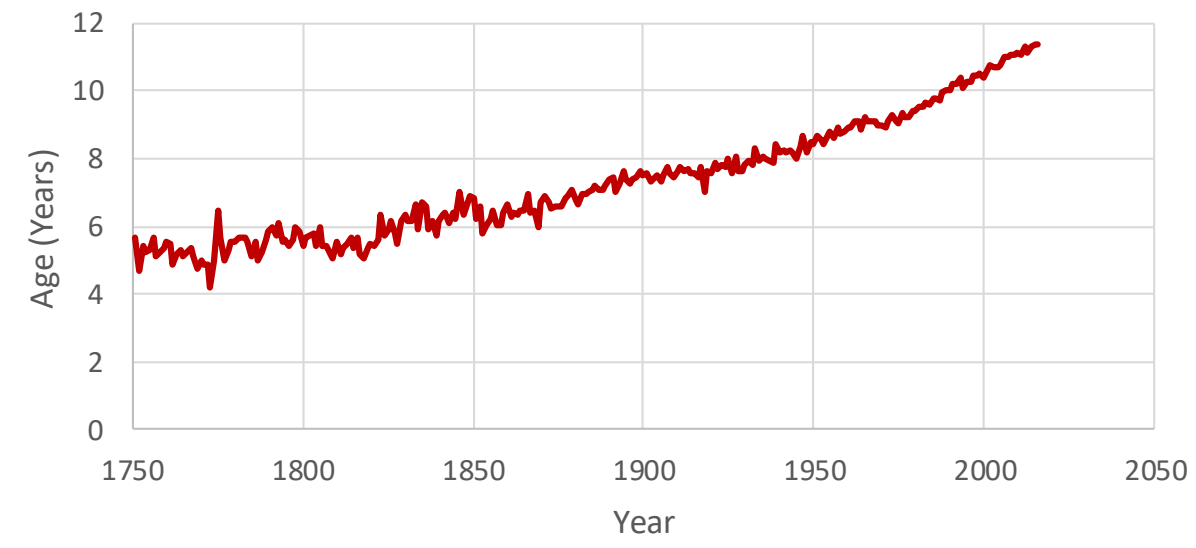

Fig. 2. Healthy Life Years Lost (HLYL) in Sweden, females (1751-2016). 
The Healthy Life Years Lost (HLYL) calculated data series is illustrated in figure 2 . The HLYL trend is slightly growing until 1850 followed with a faster grow until 11.35 years of age in 2016 after 5.69 years of age in 1751.

\section{The Logistic Model}

This classical model proposed by P. F. Verhulst in 1838 to estimate the population of France is proven to be a successful tool for long range forecasting. In his first application, Verhulst predicted the population of France for almost 100 years. Pearl and Reed used this model to predict the growth of the United States Population. Applications in other countries have also done.

The three parameter Logistic Model equation form is the following

$$
g(t)=\frac{F}{1+\left(\frac{F}{g(0)}-1\right) \exp (-b(t-T(0)))^{\prime}}
$$

Where $b$ is the trend or diffusion parameter and $F$ is the upper level of the sigmoid logistic process and $g(0)$ is the value at time $T(0)=1900$. 


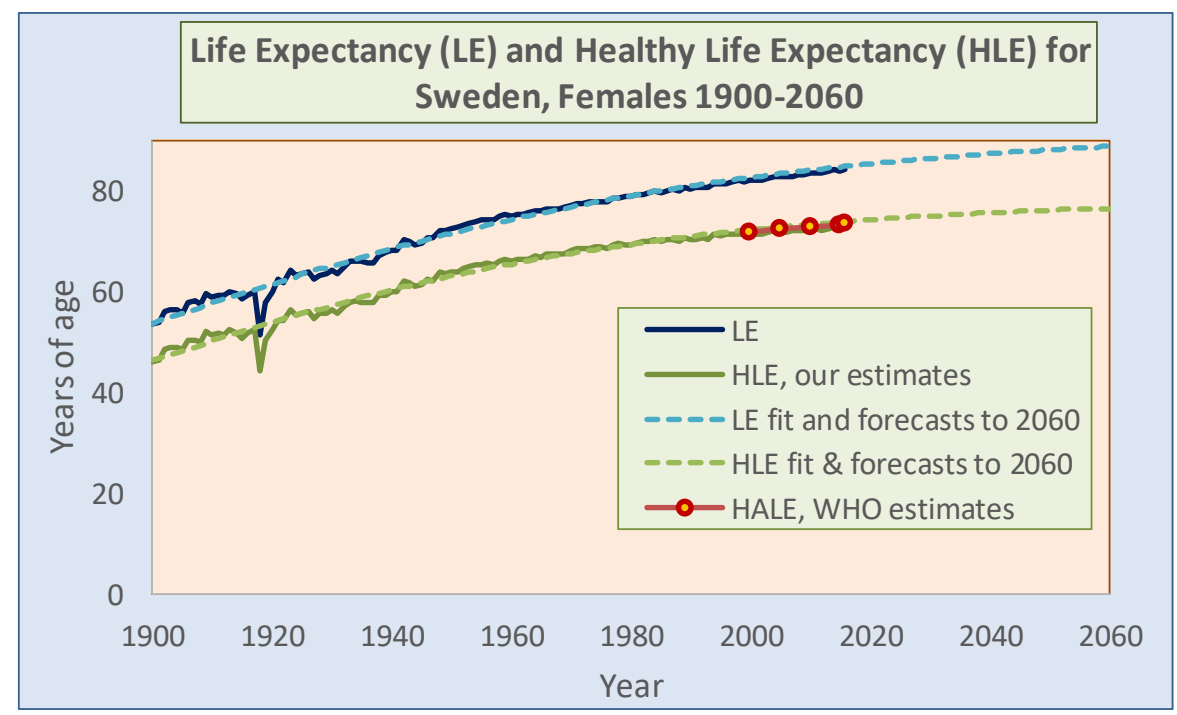

Fig. 3. Logistic model fit and forecasts to 2060 for females in Sweden.

\section{The HALE estimates and our Direct calculations}

The latest WHO estimates for Healthy Life Expectancy called HALE are provided for the years 2000, 2005, 2010, 2015 and 2016.

These estimates perfectly fit into our calculations for the HLE and the fit results by using the Logistic model.

Our HLE calculations are based on the Direct estimates from the Life Tables of the HLYL with a formula provided in recent publications (Skiadas \& Skiadas 2020a,b,c) that is:

$$
H L Y L=\max \frac{x m_{x}}{\sum_{0}^{x} m_{x}}
$$

Where $m_{x}$ is the mortality at age $x$ as provided in HMD life tables.

Then HLE=LE-HLYL. 
The Logistic model is applied to data sets for LE and HLE from our estimates from 1900 to 2016. The parameters selected appear in the following Table I.

The Healthy Life Years Lost (HLYL) are 7.40 years of age in 1900, 10.19 for 2016, 12.15 in 2060 with a maximum of $F=13.74$ years of age difference.

TABLE I. Logistic model parameters and estimates

\begin{tabular}{|c|c|c|c|c|c|}
\hline & \multicolumn{2}{|c|}{$\begin{array}{c}\text { Logistic Model } \\
\text { Parameters }\end{array}$} & \multicolumn{3}{c|}{ LE and HLE in } \\
& 1900, 2016 and 2060 \\
\hline & $\mathrm{b}$ & $\mathrm{F}$ & 1900 & 2016 & 2060 \\
\hline $\mathrm{LE}$ & 0.01820 & 92.15 & 53.70 & 84.09 & 88.70 \\
\hline HLE & 0.02096 & 78.41 & 46.30 & 73.90 & 76.55 \\
\hline $\begin{array}{c}\text { HLYL= } \\
\text { LE-HLE }\end{array}$ & & 13.74 & 7.40 & 10.19 & 12.15 \\
\hline
\end{tabular}

Table II summarizes the three healthy life expectancy estimates from WHO (HALE), our Direct estimates and from Logistic fit. All three methodologies provide close results.

TABLE II. HALE and Healthy Life Expectancy Direct estimates and Logistic fit

\begin{tabular}{|c|c|c|c|c|c|}
\hline Year & 2000 & 2005 & 2010 & 2015 & 2016 \\
\hline $\begin{array}{c}\text { WHO } \\
\text { HALE }\end{array}$ & 71.74 & 72.37 & 72.97 & 73.27 & 73.36 \\
\hline $\begin{array}{c}\text { Direct } \\
\text { HLE }\end{array}$ & 71.63 & 71.98 & 72.32 & 72.67 & 72.74 \\
\hline $\begin{array}{c}\text { Logistic } \\
\text { Fit }\end{array}$ & 72.25 & 72.81 & 73.34 & 73.81 & 73.90 \\
\hline
\end{tabular}




\section{Conclusions}

We have solved the problem of finding the HLE in the far past. The case of Sweden (1751-2016, females) with forecasts to 2060 and comparisons with HALE has explored. The selected Logistic model has a good fit while the HALE estimates from WHO compare very good to our estimates both with Direct method and the logistic fit.

\section{References}

Skiadas, C.H. and Skiadas, C. (2018). Exploring the Health State of a Population by Dynamic Modeling Methods. The Springer Series on Demographic Methods and Population Analysis 45, Springer, Chum, Switzerland. https://doi.org/10.1007/978-3-319-65142-2.

Skiadas, C.H. and Skiadas, C. (2018). Demography and Health Issues: Population Aging, Mortality and Data Analysis. The Springer Series on Demographic Methods and Population Analysis 46. Springer, Chum, Switzerland. https://doi.org/10.1007/978-3-319-76002-5.

Skiadas, C.H. and Skiadas, C. (2020). Demography of Population Health, Aging and Health Expenditures. The Springer Series on Demographic Methods and Population Analysis 50. Springer, Chum, Switzerland. https://www.springer.com/gp/book/9783030446949 , https://doi.org/10.1007/978-3-030-44695-6

Skiadas, C.H. and Skiadas, C. (2020). Relation of the Weibull Shape Parameter with the Healthy Life Years Lost Estimates: Analytical Derivation and Estimation from an Extended Life Table. In The Springer 
Healthy Life Expectancy in Sweden 9

Series on Demographic Methods and Population Analysis 50. Springer, Chum, Switzerland. https://doi.org/10.1007/978-3-030-44695-6 2

Skiadas, C.H. and Skiadas, C. (2020). Direct Healthy Life Expectancy Estimates from Life Tables with a Sullivan Extension. Bridging the Gap Between HALE and Eurostat Estimates. In The Springer Series on Demographic Methods and Population Analysis 50. Springer, Chum, Switzerland. https://doi.org/10.1007/978-3-030-44695-6 3

Sundin, J. and Willner, S. (2007). Social change and health in Sweden 250 years of politics and practice. Swedish National Institute of Public Health. 